Approved for public release; distribution is unlimited

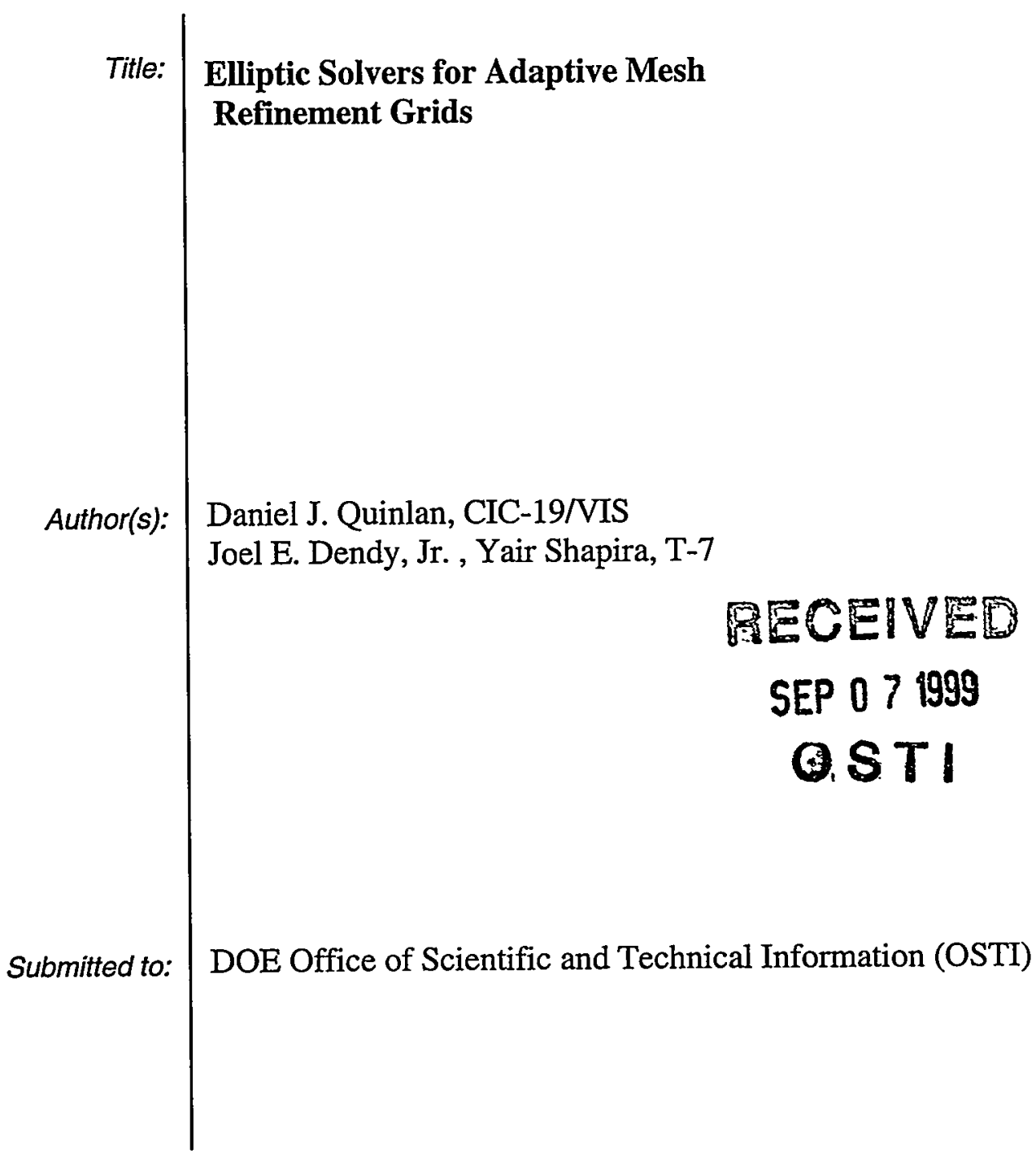

Los Alamos National Laboratory, an affirmative action/equal opportunity employer, is operated by the University of California for the U.S. Department of Energy under contract W-7405-ENG-36. By acceptance of this article, the publisher recognizes that the U.S. Government retains a nonexclusive, royaltyEner free license to publish or reproduce the published form of this contribution, or to allow others the auspices of the U.S. Department of Energy. Los Alamos National

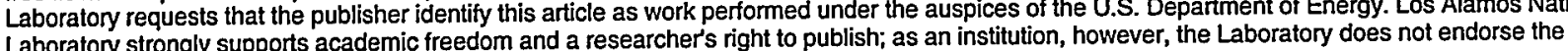
viewpoint of a publication or guarantee its technical correctness. 


\section{DISCLAIMER}

This report was prepared as an account of work sponsored by an agency of the United States Government. Neither the United States Government nor any agency thereof, nor any of their employees, make any warranty, express or implied, or assumes any legal liability or responsibility for the accuracy, completeness, or usefulness of any information, apparatus, product, or process disclosed, or represents that its use would not infringe privately owned rights. Reference herein to any specific commercial product, process, or service by trade name, trademark, manufacturer, or otherwise does not necessarily constitute or imply its endorsement, recommendation, or favoring by the United States Government or any agency thereof. The views and opinions of authors expressed herein do not necessarily state or reflect those of the United States Government or any agency thereof. 


\section{DISCLAIMER}

Portions of this document may be illegible in electronic image products. Images are produced from the best available original document. 


\title{
Elliptic Solvers for Adaptive Mesh Refinement Grids
}

\author{
Daniel J. Quinlan*, CIC/VIS, \\ J.E. Dendy, Jr., Yair Shapira, T-7
}

\begin{abstract}
We are developing multigrid methods that will efficiently solve elliptic problems with anisotropic and discontinuous coefficients on adaptive grids. The final product will be a library that provides for the simplified solution of such problems. This library will directly benefit the efforts of other Laboratory groups. The focus of this work is research on serial and parallel elliptic algorithms and the inclusion of our black-box multigrid techniques into this new setting. The approach applies the Los Alamos object-oriented class libraries that greatly simplify the development of serial and parallel adaptive mesh refinement applications.

In the final year of this LDRD, we focused on putting the software together; in particular we completed the final AMR++ library, we wrote tutorials and manuals, and we built example applications. We implemented the Fast Adaptive Composite Grid method as the principal elliptic solver. We presented results at the Overset Grid Conference and other more AMR specific conferences. We worked on optimization of serial and parallel performance and published several papers on the details of this work. Performance remains an important issue and is the subject of continuing research work.
\end{abstract}

\section{Background and Research Objectives}

Our objective was to develop multigrid methods which would efficiently solve elliptic problems with anisotropic and discontinuous coefficients on adaptive grids. The final result will be a library that provides for the simplified solution of such problems. This library will directly benefit the efforts of CIC-19 (Computer Research and Applications), EES-5 (Geoanalysis), T-3 (Fluid Dynamics), T-7 (Mathematical Modeling and Analysis), and XTM (Radiation Transport), and others. The focus of this work was research on FAC, AFAC and AFACx (described below) for serial and parallel environments and inclusion of the black box multigrid techniques developed by Dendy

*Principal Investigator, e-mail: dquinlan@lanl.gov 
into this new setting. With this library details of applications are easy to define within the scope of the A++/P++/AMR++ [LQ2,LQW,BLQ] array class libraries, and details of adaptive mesh refinement are properly isolated but available to the target application. The multigrid elliptic solvers are a natural part of the library and thus readily accessible. The use of the $\mathrm{A}++/ \mathrm{P}++/ \mathrm{AMR}++$ array classes provides portability between different architectures; that is, use of these class libraries means that one code runs efficiently on a variety of platforms.

Many important modeling problems at Los Alamos involve the solution of partial differential equations in which the coefficients are discontinuous and anisotropic, jumping by orders of magnitude across internal interfaces. These problems also involve mixed length scales which require either uniformly fine meshes or adaptively refined meshes. Examples include the modeling of petroleum reservoirs, semiconductor devices, contaminant transport, and nuclear reactors; these are all activities in which the Laboratory is involved. More specifically, the modeling of combustion, for example, involves the modeling of infrared radiation transfer, heat conduction, and chemical reaction-diffusion equations; each of these exhibit anisotropic and discontinuous coefficients or mixed length scales or both. The same situation occurs in the modeling of impurity doping and of charge transport in the study of semiconductor devices. The point of mentioning these separate research efforts is to emphasize that while many areas of work at LANL require adaptive mesh refinement, the traditional high cost in developing these techniques for each new architecture has minimized their use.

Previous work at LANL by Dendy resulted in the Black Box multigrid software. We have extended this work to incorporate adaptive mesh refinement applications. Previous work by Quinlan successfully used $\mathrm{C}++$ object-oriented techniques to simplify development and research on serial and parallel adaptive mesh refinement algorithms. Quinlan's work with Steve McCormick led to the development of AFAC and AFACx [MQ,LQ1] algorithms, which provided enhanced parallelism of AMR elliptic solvers through improved parallel algorithms, very different in design from their serial counterparts. But AFAC and AFACx, though understood theoretically, had not previously been applied to the same complex applications for which Black Box MG had been developed. This research provided an opportunity to mix our talents and to additionally provide a library of the resulting "Black-Box-AMR" methods. The library of elliptic solvers, though not the primary focus of our research, makes these techniques available to other scientists wishing to develop serial and parallel applications, using Quinlan's A++/P++/AMR ++ libraries. For our own use, the library simplifies our identification of relevant research approaches and provides intuition for further work. 
The development of black box multigrid solvers can be motivated by the standard difference approximation to the simplest second order differential equation in one space dimension. In this case, the coarse grid can be formed from every other grid point of the fine grid, interpolation can be derived from the difference equation, and the coarse grid can be formed by employing a variational (Galerkin) principle. Employing this process recursively results in a version of cyclic reduction. In two space dimensions, approximations to this process must be employed to keep the stencil of the operator from growing without bound on the coarser grids. The basic principles remain the same, however: the use of operator induced interpolation and the formation of the coarse grid equations via a variational principle. In more than one space dimension, of course, the choice of a good smoothing scheme is nontrivial. Current black box software provides for the solution of nonsymmetric equations in two and three dimensions on logically rectangular and logically cuboid grids.

The fast adaptive composite grid method (FAC) is an algorithm that uses uniform grids, both global and local, to solve partial differential equations. This method is highly efficient on scalar and single processor vector computers, due to its effective use of uniform grids and due to the multiple levels of resolution of the solution. On distributed memory multiprocessors, such methods benefit from the creation of multiple isolated refinement regions, which may be effectively treated in parallel. However, FAC suffers from the sequential treatment of the levels of refinement in each region. Specifically, the finer levels must wait to be processed until the coarse-level approximations have been computed and passed to them; conversely, the coarser levels must wait until the finer level approximations have been computed and used to correct their equations. The algorithm is well suited to serial machines and can be parallelized, but its parallelization is restricted to the parallelization of the processing on each refinement level, because of the synchronous processing of the refinement levels.

The asynchronous fast adaptive composite method (AFAC) eliminates this bottleneck of parallelism. Through a simple mechanism used to reduce inter-level dependence, individual refinement levels can be processed by AFAC in parallel. The result is that the convergence rate of AFAC is the square root of that for FAC. Therefore, since both AFAC and FAC have roughly the same number of floating point operations, $\mathrm{AFAC}$ requires twice the serial computational time as FAC. However, AFAC may be much more efficiently parallelized. The most crucial factor for the evaluation of both algorithms is the different computation/communication cost ratios for these architectures. It has been shown that, under many circumstances, AFAC is superior to FAC in a parallel 
environment, since AFAC generates fewer total messages than FAC. The difference in the number of messages can be approximately three orders of magnitude (or more) on a moderate 64 processor parallel environment, but is dependent on the adaptively refined grid chosen. AFACx is an enhanced version of AFAC, which modifies the solution process to permit the use of simple relaxation instead of approximate solutions on each refinement level. The result is a significantly cheaper adaptive mesh refinement solver exhibiting significantly reduced communication in the parallel environment. In all cases, the convergence rates of AFAC and AFACx are identical.

\section{Importance to LANL's Science and Technology Base and National R\&D Needs}

The ultimate objective of this work is to provide better modeling techniques for scientists working on problems listed in the introduction. The methods that are needed for these problems are too complicated to be developed over and over again for each new application. The design of elliptic solvers to handle discontinuous and anisotropic coefficients on adaptively refined grids is new and will make available a tool for others to use. The simultaneous development of the associated parallel algorithm work provides even greater impact, and just in time for use on LANL's growing collection of parallel computers. Providing both within a similar framework, using the AMR++ library and the serial and parallel $(\mathrm{A}++/ \mathrm{P}++)$ array class libraries, will make the resulting work incredibly useful to LANL scientists.

\section{Scientific Approach and Accomplishments}

Long before this LDRD, Dendy had developed a multigrid method which employed locally refined grids. [D1, D3] This method was robust for problems with anisotropic and discontinuous coefficients, but the locally refined grids were static. The resulting code was written in FORTRAN, the language of choice in those halcyon days. It employed a mocked up version of pointers, but the daunting task of making these pseudo-pointers dynamic led to the admission of defeat.

Today object-oriented programming is the obvious tool for this problem. The class libraries that Quinlan has written make it possible to keep the details of the dynamically refined local grids in the background, where they belong. The development of these tools for serial and parallel adaptive mesh refinement, will simplify future work on algorithms for difficult elliptic equations. Previous attempts using more traditional approaches have routinely failed to support more than the algorithmic development specific to relatively simple elliptic equations; our approach allows for much greater generality and can be applied to much more complicated equations. 
Object-oriented programming does not remove the basic algorithmic difficulty: When multigrid is employed, at some stage the coarse grids become so coarse that they must provide a homogenized version of the details that are present on finer grids. On logically rectangular grids, we have long known how to provide this homogenization. With the presence of locally refined grids, homogenization becomes more difficult. One solution was provided in [D1, D3]; Shapira [11] derived new solutions. Before this LDRD, FAC/AFAC/AFACx methods had only been applied to problems with smooth coefficients and had only employed simple interpolation, residual restriction, and relaxation; the work in this LDRD extends their application to problems with discontinuous coefficients, through the use of "black box multigrid" ideas.

Development of the AMR++ library proved to be the chief bottleneck in this project. A simple application of Hofstadter's Law --- "Every project takes three times long as anticipated, even when Hofstadter's Law is taken into account" --- might have allowed us to predict this situation. The longer than anticipated development time for AMR++ prevented us from completing all that we had hoped to achieve. We plan to continue work on this project, with the following goals:

1) Inclusion of semicoarsening within the elliptic solves on each refinement level. This feature offers many advantages, especially if it is employed in the definition of the composite grid operator. However, multiblock grids are an unavoidable part of the adaptive grid at each level of refinement, and their presence complicates the semicoarsening approach. 2) Improvements to AFAC. AFAC introduces parallelism previously not present in the FAC algorithm, with a resulting improvement of parallel performance by more than an order of magnitude on a moderate number of processors [LQ1]. However, the evaluation of the residual in AFAC, using the composite grid operator, remains synchronous. Improved parallel performance would result from algorithmic improvement to the step which evaluates the residual using the composite grid operator. This work would largely complete the AFAC/AFACx work and make high performance parallel elliptic solvers for adaptively refined grid more practical. 3) Extension of elliptic solver technology for adaptively refined grids to handle overlapping grids. The nature of the unstructured overlap present in the overlapping grid structure greatly complicates the solution process for elliptic equations with anisotropic and discontinuous coefficients. Success in this area would provide a much-needed tool for the extension of overlapping grids to more complex applications. 


\section{Publications}

1. Bassetti, F., Davis, K., Quinlan, D., "OVERTURE: An Object-Oriented Framework for High Performance Scientific Computing", Proceedings of the Super Computing'98 Conference (accepted), Orlando, Florida, November 13-16 1998

2. Bassetti, F., Davis, K., Quinlan, D., "Optimizing Transformations of Stencil Operations for Parallel Object-Oriented Scientific Frameworks on Cache-Based Architectures", Proceedings of the ISCOPE'98 Conference (accepted), Santa Fe, New Mexico, Dec 13-16 1998

3. Bassetti, F., Davis, K., Quinlan, D., "Loop Transformations for Performance and Message Latency Hiding in Parallel Object-Oriented Frameworks", Proceedings of the PDPTA'98 Conference, Las Vegas, Nevada, July 13-16 1998

4. Bassetti, F., Davis, K., Quinlan, D., "Temporal Locality Optimizations for Stencil Operations within Parallel Object-Oriented Scientific Frameworks on Cache-Based Architectures", Proceedings of the PDCS'98 Conference, Las Vegas, Nevada, July 18-23 1998

5. Quinlan, D., "AMR++: Object-Oriented Design for Adaptive Mesh Refinement", Proceedings of the HPC'98 Conference, Boston, Mass. April 5-9, 1998

6. Bassetti, F., Davis, K., Quinlan, D., "Toward FORTRAN 77 Performance From Object-Oriented C++ Scientific Frameworks", Proceedings of the HPC'98 Conference, Boston, Mass. April 5-9, 1998

7. Bassetti, F., Davis, K., Quinlan, D., "C++ Expression Templates Performance Issues in Scientific Computing", Proceedings of the IPPS Conference, Boston, Mass. March, 1998

8. Brown, D., Henshaw, W. Quinlan, D., "Overture: An Object-Oriented Framework for Solving Partial Differential Equations", Proceedings of the first International Scientific Computing in Object-Oriented, Parallel Environments (ISCOPE) Conference, Marina del Rey, California, Dec, 1997

9. Bassetti, F., Davis, K., Quinlan, D., "A Comparison of Performance-enhancing Strategies for Parallel Numerical Object-Oriented Frameworks", Proceedings of the first International Scientific Computing in Object-Oriented, Parallel Environments (ISCOPE) Conference, Marina del Rey, California, Dec, 1997

10. Shapira, Y., "Analysis of Matrix-dependent Multigid Algorithms, Num. Lin. Alg. Applic. 5, 165-201 (1998).

11. Shapira, Y., "Multigrid for Locally Refined Meshes, to appear in SIAM J. Sci. Comput. 


\section{References}

[1] [ABDP] R. E. Alcouffe, A. Brandt, J.E. Dendy, Jr., and J. W. Painter, The Multi-Grid Method for the Diffusion Equation with Strongly Discontinuous Coefficients, S SISSC 2, No. 4, 430-454 (1981).

[2] [D1] J.E. Dendy, Jr., Local Grid Refinement in the Multigrid Methods Trans. A.N.S., Vol. 38, 342-343 (1981).

[3] [D2] J.E. Dendy, Jr., Black Box Multigrid, Proceedings from Symposium on Multigrid Methods, Moffett Field, California, October 19-22, 1981, 249-274, also J. Comp. Phys. 48, No. 3 (1982) 366-386.

[4] [D3] J.E. Dendy, Jr., A Priori Local Grid Refinement in the Multigrid Method, Elliptic Problem Solvers II, G. Birkhoff, A. Schoenstadt, eds., Academic Press, New York, 1984.

[5] [D4] J.E. Dendy, Jr., Two Multigrid Methods for Three-Dimensional Problems with Discontinuous and Anisotropic Coefficients, SIAM J. Sci. Stat. Comp. 8, No. 2, 673-685 (1987).

[6] [DMRRS] S. F. McCormick, J. W. Ruge, T. F. Russell, S. Schaffer, Multigrid Methods for Three-Dimensional Petroleum Reservoir Simulation, Proceedings of Tenth S. P. E. Symposium on Reservoir Simulation, February 6-8, 1989, Houston, Texas, pp. 19-25 (1989).

[7] [MDHW] J. E. Morel, J.E. Dendy, Jr., M. Hall, S. White, A Cell-Centered Lagrangian Mesh Diffusion Differencing Scheme, J. Comp. Phys.. 103(1992), 286-299.

[8] [DIR] M. P. Ida, J. M. Rutledge, "A Semi-Coarsening Multigrid Algorithm for SIMD Machines," SIAM J. Sci. Stat. Comp. 13(1992), 1460-1469.

[9] [MDW] J. E. Morel, J.E. Dendy, Jr., and T. A. Wareing, Diffusion-Accelerated Solution of the Two-Dimensional Sn Equations with Bilinear-Discontinuous Differencing, Nucl. Sci. Eng. 115(1993), 304-319.

[10] [BDS] R.G. Brickner, J.E. Dendy, Jr., and W.H. Spangeburg, CRATA with Thinking Machines for CMSSL.

[11] [BLQ] Balsara, D., Lemke, M., Quinlan, D., "AMR++, a C++ ObjectOriented Class Library for Parallel Adaptive Refinement Fluid Dynamics Applications, Proceedings of American Society of Mechanical Engineers, Winter Annual Meeting, Annahiem, CA, November 8-13, Adaptive, Multilevel and Hierarchical Computational Strategies , AMD-Vol. 157, pg. 413-433, 1992 
96310

[12] [LQW] Lemke, M., Quintan, D., Witsch, K., "An Object Oriented Approach for Parallel Self Adaptive Mesh Refinement on Block Structured Grids", Proceedings of the 9th GAMM-Seminar Kiel, Notes on Numerical Fluid Mechanics, Viewer, Germany, 1993.

[13] [MQ] McCormick, S., Quintan, D., "Asynchronous Multilevel Adaptive Methods for Solving Partial Differential Equations on Multiprocessors: Performance Results," Parallel Computing, Vol. 12, 1989, pp. 145-156.

[14] [LQ1] Lemke, M., Quintan, D., "Fast Adaptive Composite Grid Methods on Distributed Parallel Architectures", Proceedings of the Fifth Copper Mountain Conference on Multigrid Methods, Copper Mountain, CO, April 1991. Also in Communications in Applied Numerical Methods, Vol. 8, No. 9, pg. 609-619, Sept 1992

[15] [LQ2] Lemke, M., Quinlan, D.,"P++, a C++ Virtual Shared Grids Based Programming Environment for Architecture-Independent Development of Structured Grid Applications", accepted for CONPAR/VAPP V, September 1992, Lyon, France; to be published in Lecture Notes in Computer Science, Springer Verlag, September 1992.

8 
refined grid after update refinement
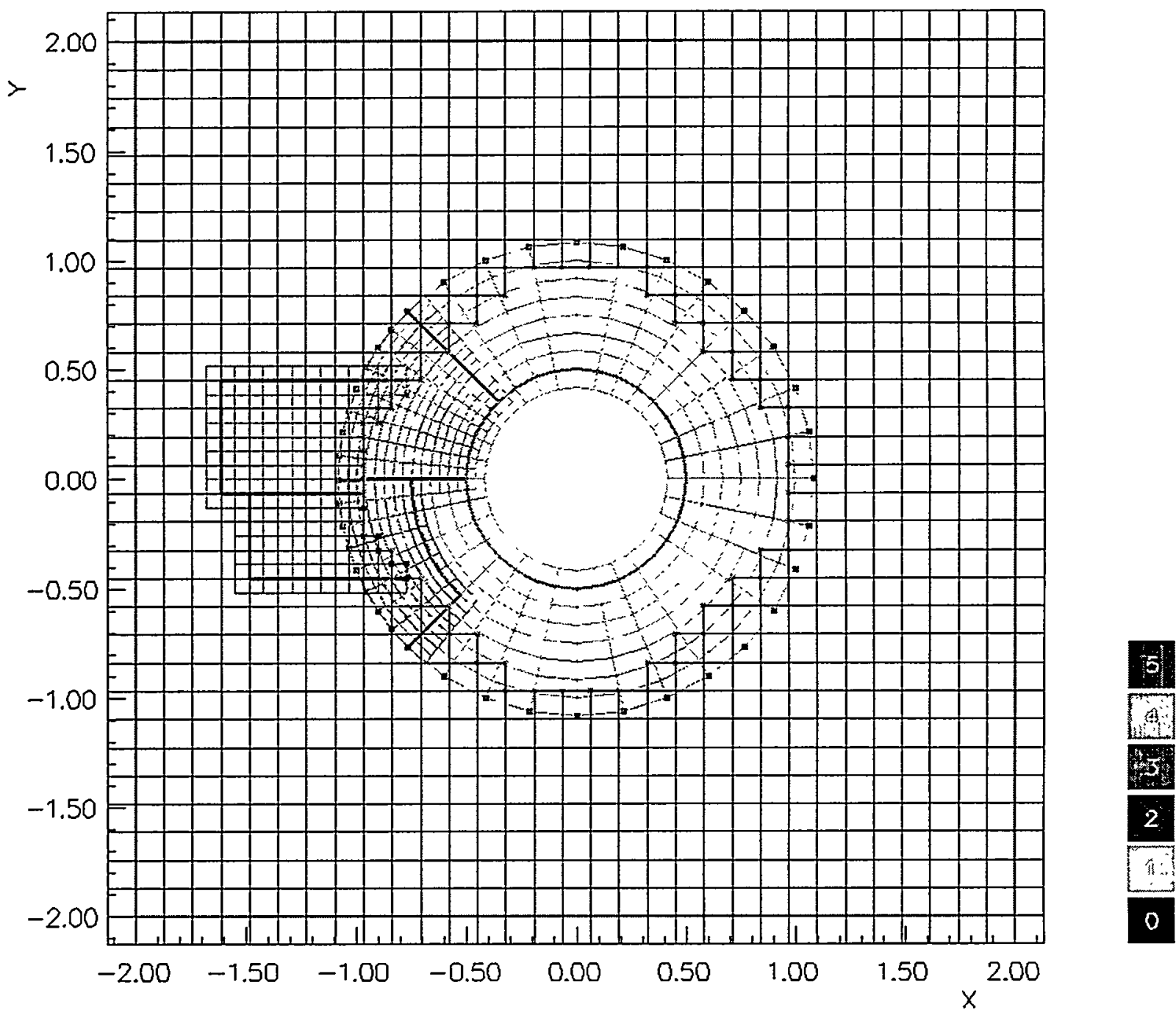

Figure 1: Adaptive Overlapping Grid 


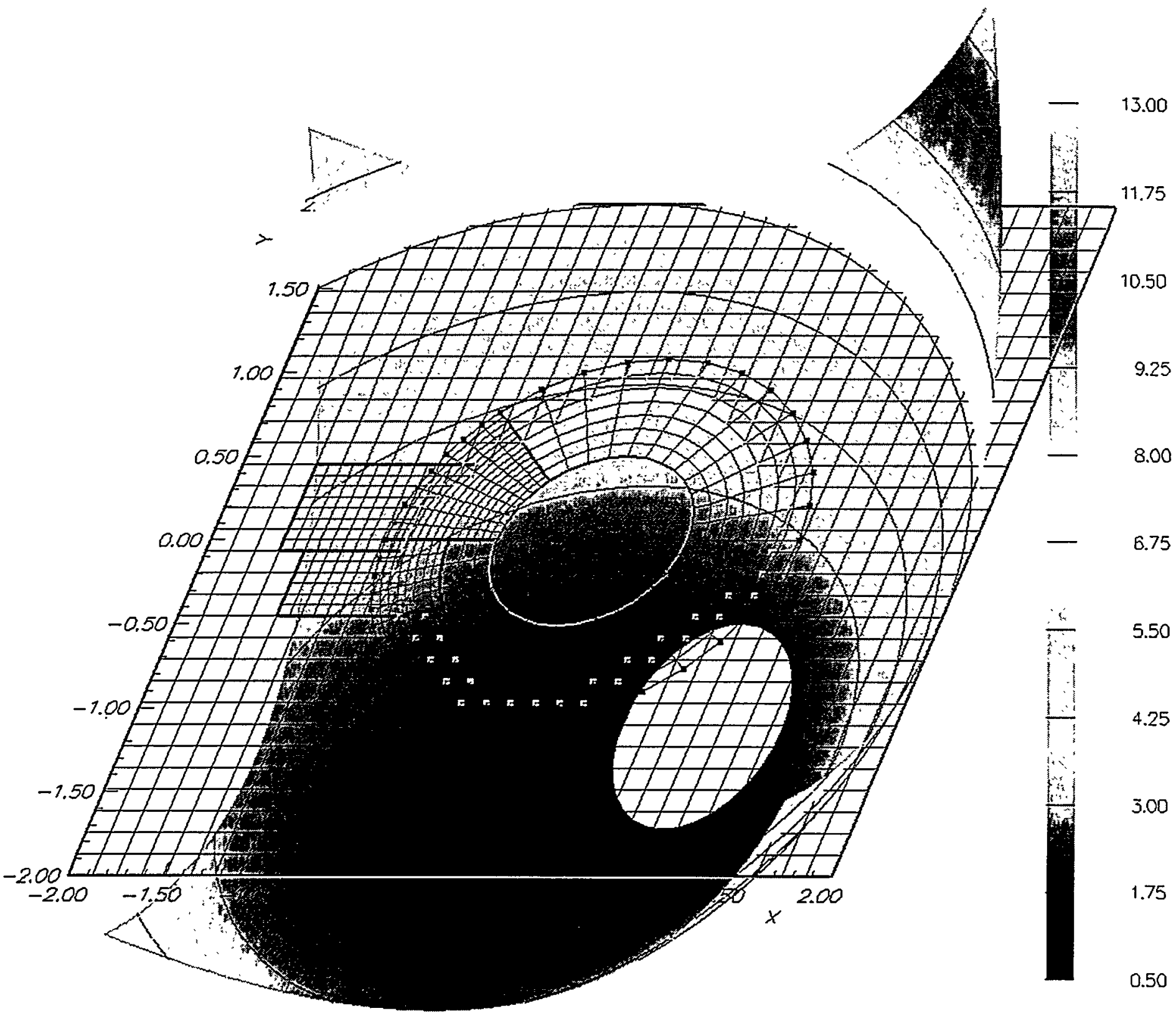

Figure 2: Solution on Adaptive Overlapping Grid 


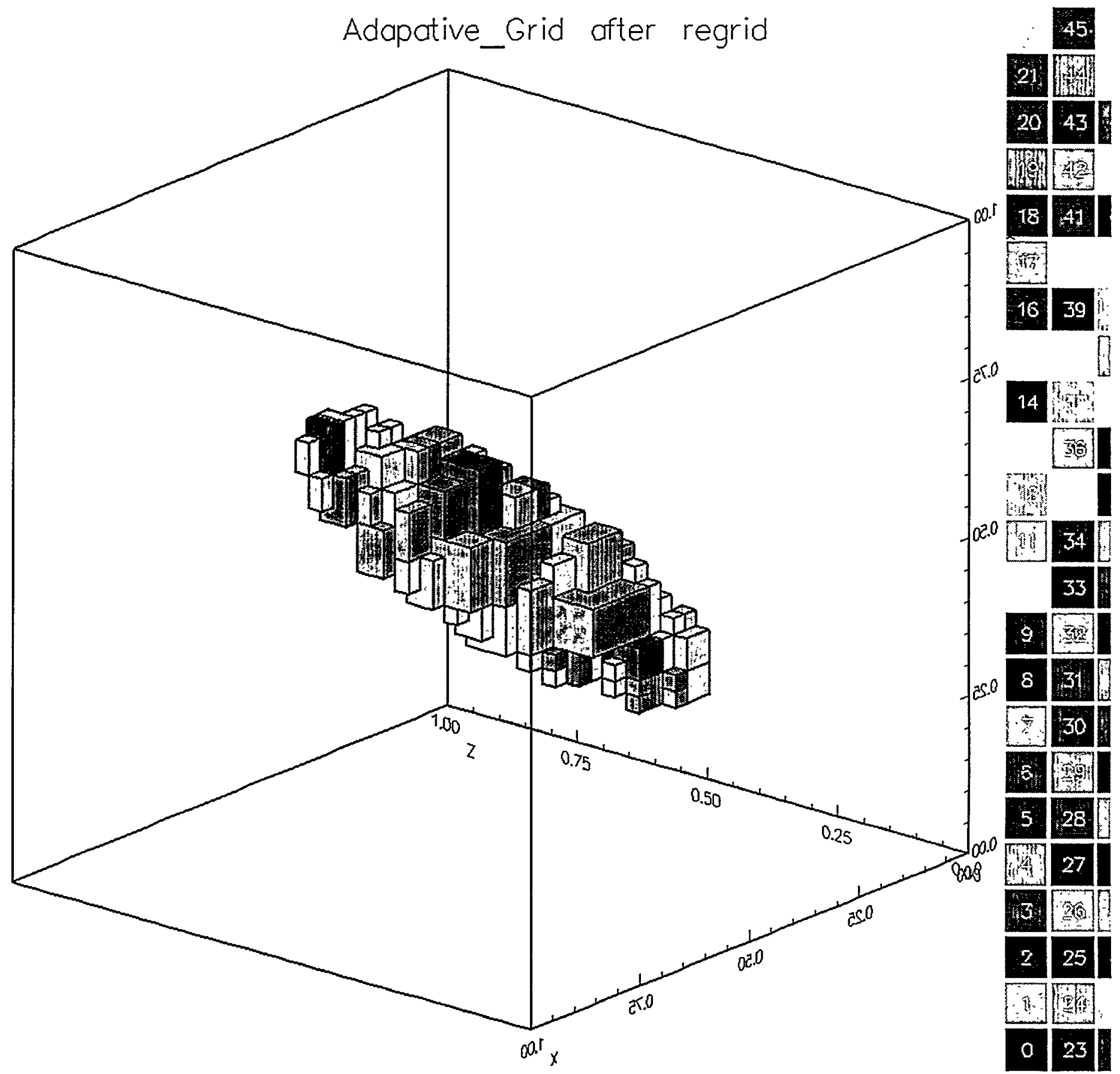

Figure 3: Three-dimensional Grid With Refinement 